\title{
LAA closure in pre-dialytic and dialytic patients: A case report and mini review of literature
}

\author{
Mohamed Abdirashid*, Fabrizio Ugo, Mario Matta, Chiara Cavallino, Ludovica Maltese, Danilo Reale, Virginia Di Ruocco and Francesco \\ Rametta \\ Department of Cardiology, Sant'Andrea Hospital, Vercelli Italy
}

\begin{abstract}
Atrial fibrillation is the most common arrhythmia and it increases the risk of stroke with it's potentially disabling burden. More than $90 \%$ of thrombi originates from the left atrial appendage (LAA) and so far, oral anticoagulation (OAC) with vitamin $\mathrm{K}$ antagonist (VKA) or with non-vitamin $\mathrm{K}$ oral anticoagulants (NOACs) is the standard care for stroke prevention. Unfortunately, different subsets of patients have contra-indications to or a failure of OACs that represents an indication to left atrial appendage closure (LAAC). We present a case of a successful LAAC in an 75-year-old gentlemen with permanent AF and a history of dialytic-CKD, referred to our institution due to multiple GI bleeding from colic diverticulosis and an episode of post-traumatic intra-articular knee bleeding on OAC with warfarin. OAC in CKD patients, in particular in end stage and pre-dialytic patients, represents a therapeutic challenge since these patients are burdened by an increased ischemic and hemorrhagic risk per se, with a labile INR on VKA and frequent contra-indications to NOACs due to low glomerular filtration rate (GFR). For this reason, LAAC fulfils an unmet therapeutic need especially in CKD patients with a rich literature that demonstrates its feasibility, safety and benefits in patients with chronic nephropathy. Moreover, the recent development of contrast free techniques, thanks to the use of 3D trans-esophageal echocardiography both in the pre-procedural evaluation of the LAA morphology and during the intervention, represents a promising prospect in this high risk sub-population.
\end{abstract}

\section{Introduction}

Atrial fibrillation is the most common arrhythmia, affecting $2 \%$ of the general population [1]. Moreover, $20-30 \%$ of ischemic strokes are caused by cardiogenic thromboembolism, mainly due to atrial fibrillation, representing a potentially disabling complication of this arrhythmia [2-4]. In patients with non-valvular AF, $>90 \%$ of thrombi originates from the left atrial appendage (LAA) [5]. The use of VKA or NOACs to prevent ischemic events is recommended in patients with high ischemic risk, assessed with CHADS2VASc Score. However, anticoagulation therapy can in some subsets of patients cause haemorrhagic events that can outweighs its potential benefits. Therefore, it is recommended to assess individual bleeding risk with HAS-BLED score to identify those patients in which the risk of the therapy outweigh its benefits [3].

Unfortunately, it is a frequent occurrence to have an increased ischemic and haemorrhagic risk especially in elderly patients since most of the risk factors concur to increase the incidence of both events. For this subset of patients, the left atrial appendage closure (LAAC) represents an interventional alternative and was proven to be effective and safe in high-risk patients. Another indication for LAAC is when there is a failure of anticoagulation therapy, with ischemic events despite adequate therapy [6].

\section{LAAC indications}

According to the 2020 EHRA/EAPCI consensus we can summarise the indication for LAAC as it follows:

Patients with a contraindication for OAC:

- $\mathrm{HASBLED} \geq 3$

- Elevated bleeding risk outside HASBLED score (e.g. tumor, thrombocytopaenia)
- Need for prolonged or repetitive triple therapy (e.g. Severe CAD and stenting)

- Renal failure (severe) as contraindication to NOAC

- Severe side effect under vitamin K antagonist

- Patients with an elevated bleeding risk under chronic OAC (e.g. post intracranial bleeding)

- Non-compliant patients (unwilling or unable to take OAC)

- OAC not efficient ("stroke on OAC")

- Patients with non-valvular AF who are eligible for chronic longterm OAC only if they refuse OAC despite explanation.

\section{The case}

A 75-year-old gentlemen was admitted in the nephrology department of our hospital for worsening of the renal function due to an infection of $C$. difficile. This patient had a pre-dialytic CKD since he had a nephrectomy in 2003 due to a papillary carcinoma that became dialytic during this hospitalisation. Moreover, he had a history of ischaemic heart disease with an $\mathrm{EF}$ of $48 \%$ due to a previous myocardial infarction treated with primary PCI with the implantation of a DES on RCA and a permanent atrial fibrillation (CHADSVASC 5, HASBLED 5). Moreover, the patient had multiple haemorrhagic events before the

*Correspondence to: Mohamed Abdirashid, Department of Cardiology, Sant’Andrea Hospital, Vercelli Italy, E-mail: baashi90@gmail.com

Key words: atrial fibrillation, left atrial appendage, chronic kidney disease, dialysis Received: March 18, 2021; Accepted: March 30, 2021; Published: April 02, 2021 
onset of the AF, mainly from GI for a history of colic diverticulosis and an episode of post-traumatic intra-articular knee bleeding on $\mathrm{OAC}$ with warfarin. After a careful consideration of the ischaemic and haemorrhagic profile of the patient and following a multidisciplinary discussion, the patient was referred for LAA closure.

Trans-esophageal echocardiography (TEE) was performed before the procedure revealing a "cactus" morphology of the LAA.

A low-contrast technique was employed for percutaneous LAA closure using a LAmbre 22/28 mm (LifeTech Scientific) device, which was successfully deployed during TEE guidance, using a low dose of contrast dye (Figures. 1-4 and Videos. 1-8). The patient's postoperative course was uneventful, and he was successfully discharged on acetylsalicylic acid active treatment alone.

At the 1-month TEE check, the device was normally positioned, no residual leak was detected, and no thrombi were found on the atrial side of the implanted device. The patient did not suffer any ischemic or thromboembolic event, nor hemorrhagic complications.

\section{OAC in CKD and haemodialytic patients}

AF patients with end-stage renal disease or on haemodialysis treatment represent a population that poses a great challenge to the clinician: on the one hand, they are taxed with a high event rate including embolic stroke; on the other hand, most NOACs are contraindicated $[7,8]$. Furthermore, these patients usually have a labile INR on VKA for reasons not completely explained by their low protein, high vegetable diet $[9,10]$. Moreover, a strong association between the use of warfarin and the development of calcifilaxis has been observed, in some studies with an odd ratio of 11 [11]. To conclude, end stage CKD and pre-dialytic patients with AF represents a "therapeutic dilemma" and an unmet need that should be satisfied by LAAC [6]. However, this procedure is often underused because it is wrongly perceived as risky and unsafe in CKD patients.

\section{LAAC in CKD patients}

The prevalence of CKD is high in AF patients, and even without a high CHADSVASc score, CKD is an independent risk factor for stroke [12]. Moreover, CKD increases the risk of bleeding, especially when patients receive OAC [7].

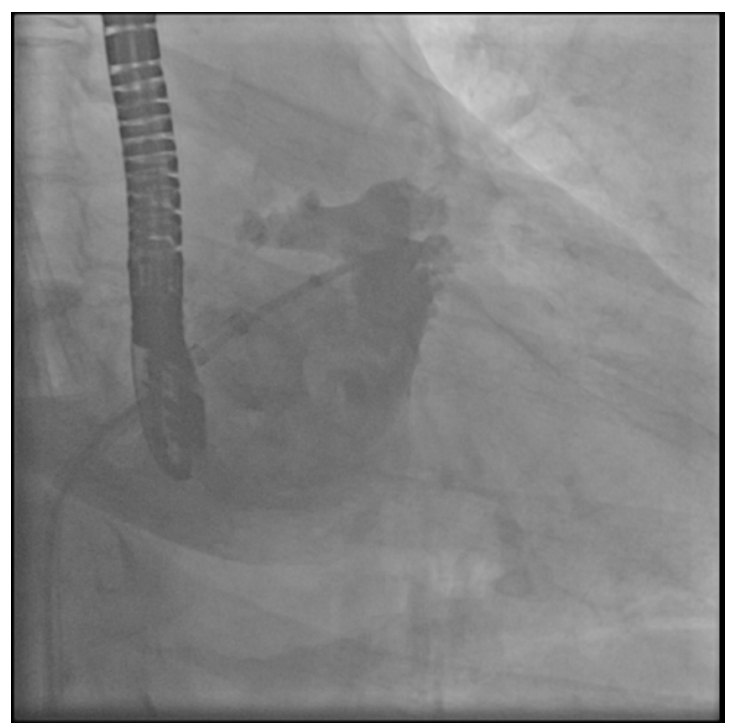

Figure 1. LAA angiography before implantation

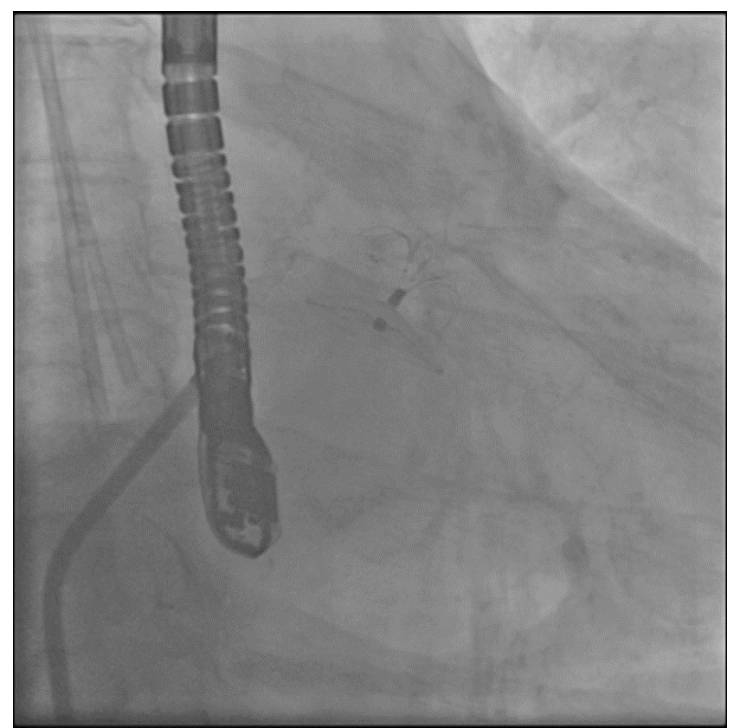

Figure 2. LAA angiography after device release

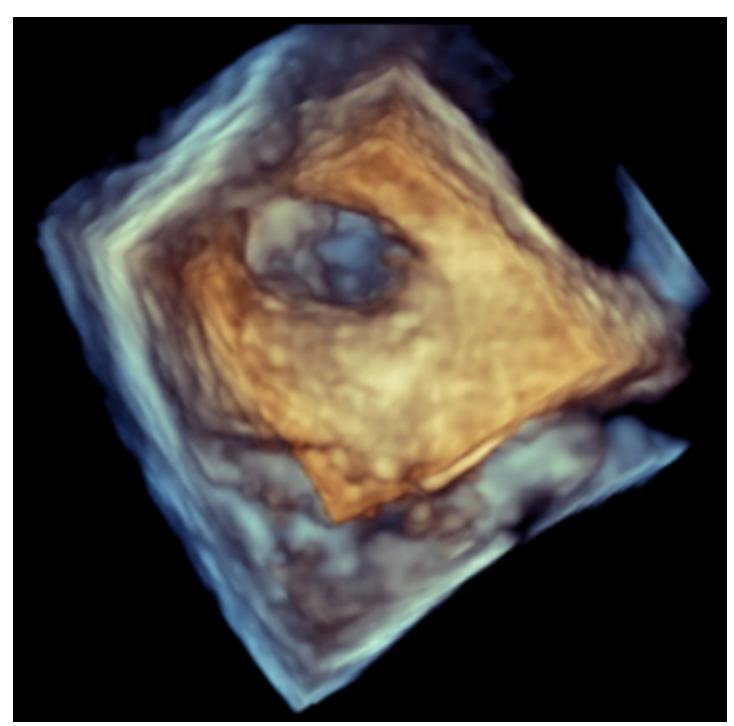

Figure 3. 3D echocardiography of LAA before device implantation

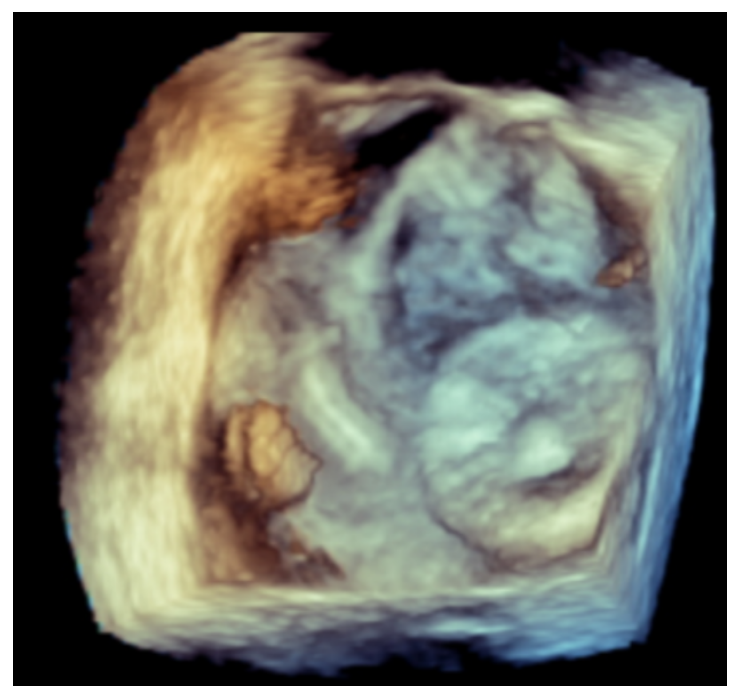

Figure 4. 3D echocardiography of LAA after device implantation 
For the aforementioned reasons LAAC should be the logical choice of treatment in this subpopulation. Nonetheless, many studies on LAAC showed a consistently high implantation success with low complications rates in all stages of renal function with a comparably low stroke rates in all groups [13-16]. However, some issues deter some clinicians from referring their patients to LAAC:

1. Contrast induced AKI in patients with impaired renal function due to

a. Contrast during the procedure

b. Contrast during the evaluation of the LAA before the procedure

\section{Procedural complications in patients with multiple comorbidities}

Post-procedural worsening of renal function may have a negative impact on clinical outcome, therefore, the role of AKI during LAAC has been studied although, evidence remains limited. Furthermore, this complication is particularly important in LAAC patients since it has been reported that more than $1 / 3$ of all patients undergoing this intervention has CKD [17]. Contrast volume (CV) and CV/eGFR ratio seems to play a critical role and it is intuitive to assume that limiting the amount of contrast volume may be a key factor [14]. Moreover, it has been demonstrated recently that contrast free TOE guided LAAC is both safe and effective and it could be a useful approach in patients with chronic renal failure $[18,19]$.

TOE in general and 3D-TOE in particular has a pivotal role in reducing the need for contrast not only during the procedure, but also during the pre-procedure evaluation possibly replacing CT with contrast. In fact, CT was almost mandatory since it was superior to cardiac angiogram in evaluating LAA measures during the workup, but 3D-TOE measures proved to have a high correlation with CT [20].

Additionally, the left atrial appendage undergoes a significant change following the volume load that is performed during the procedure to assist the implantation. Moreover, some authors suggest the need to use the same instrument both in the evaluating and procedural phase, and since TEE is the only method that can fulfil the aforementioned necessities, they support its use in all LAAC settings.

\section{Conclusion}

To conclude, LAAC in CKD patients is a safe and effective intervention that can fulfil a therapeutic unmet need in a high-risk population, especially if performed with a contrast-free TEE guided protocol.

\section{References}

1. Go AS, Hylek EM, Phillips KA, Chang Y, Henault LE, et al. (2001) Prevalence of diagnosed atrial fibrillation in adults: national implications for rhythm management and stroke prevention: The Anticoagulation and risk factors in atrial fibrillation (ATRIA) study. JAMA 285: 2370-2375. [Crossref]

2. Benjamin EJ, Muntner P, Alonso A, Bittencourt MS, Callaway CW, et al. (2019) Heart disease and stroke statistics-2019 update: A report from the American Heart Association. Circulation 139: e56-e528. [Crossref]

3. Hindricks G, Potpara T, Dagres N, Arbelo E, Bax JJ, et al. (2021) 2020 ESC Guidelines for the diagnosis and management of atrial fibrillation developed in collaboration with the European Association for Cardio-Thoracic Surgery (EACTS). Eur Heart J 42: 373498. [Crossref]
4. January CT, Wann LS, Calkins H, Chen LY, Cigarroa JE, et al. (2019) 2019 AHA ACC/HRS focused update of the $2014 \mathrm{AHA} / \mathrm{ACC} / \mathrm{HRS}$ guideline for the management of patients with atrial fibrillation: A Report of the American College of Cardiology/ American Heart Association Task Force on Clinical Practice Guidelines and the Hear Rhythm Society in collaboration with the society of Thoracic Surgeons. Circulation 140: e125-e151. [Crossref]

5. Pisters R, Lane DA, Nieuwlaat R, de Vos CB, Crijns HJ, et al. (2010) A novel userfriendly score (HAS-BLED) to assess 1-year risk of major bleeding in patients with atrial fibrillation: The Euro Heart Survey. Chest 138: 1093-1100. [Crossref]

6. Glikson M, Wolff R, Hindricks G, Mandrola J, Camm AJ, et al. (2020) EHRA/EAPCI expert consensus statement on catheter-based left atrial appendage occlusion - an update. EuroIntervention 15: 1133-1180. [Crossref]

7. Olesen JB, Lip GY, Kamper AL, Hommel K, Kober L, et al. (2012) Stroke and bleeding in atrial fibrillation with chronic kidney disease. $N$ Engl J Med 367: 625-635. [Crossref]

8. Piccini JP, Stevens SR, Chang Y, Singer DE, Lokhnygina Y, et al. (2013) Renal dysfunction as a predictor of stroke and systemic embolism in patients with nonvalvular atrial fibrillation: validation of the R(2)CHADS(2) index in the ROCKET $\mathrm{AF}$ (Rivaroxaban Once-daily, oral, direct factor Xa inhibition Compared with vitamin $\mathrm{K}$ antagonism for prevention of stroke and Embolism Trial in Atrial Fibrillation) and ATRIA (Anticoagulation and Risk factors In Atrial fibrillation) study cohorts. Circulation 127: 224-232. [Crossref]

9. Yang F, Hellyer JA, Than C, Ullal AJ, Kaiser DW, et al. (2017) Warfarin utilisation and anticoagulation control in patients with atrial fibrillation and chronic kidney disease. Heart 103: 818-826. [Crossref]

10. Kleinow ME, Garwood CL, Clemente JL, Whittaker P (2011) Effect of chronic kidney disease on warfarin management in a pharmacist-managed anticoagulation clinic. $J$ Manag Care Pharm 17: 523-530. [Crossref]

11. Cucchiari D, Torregrosa JV (2018) Calciphylaxis in patients with chronic kidney disease: A disease which is still bewildering and potentially fatal. Nefrologia 38: 579586. [Crossref]

12. Bonde AN, Lip GY, Kamper AL, Hansen PR, Lamberts M, et al. (2014) Net clinical benefit of antithrombotic therapy in patients with atrial fibrillation and chronic kidney disease: a nationwide observational cohort study. J Am Coll Cardiol 64: 2471-2482. [Crossref]

13. Boersma LV, Schmidt B, Betts TR, Sievert H, Tamburino C, et al. (2016) Implant success and safety of left atrial appendage closure with the WATCHMAN device: periprocedural outcomes from the EWOLUTION registry. Eur Heart J 37: 2465-2474. [Crossref]

14. Sedaghat A, Vij V, Streit SR, Schrickel JW, Al-Kassou B, et al. (2020) Incidence, predictors, and relevance of acute kidney injury in patients undergoing left atrial appendage closure with Amplatzer occluders: A multicentre observational study. Clin Res Cardiol 109: 444-453. [Crossref]

15. Xue X, Jiang L, Duenninger E, Muenzel M, Guan S, et al. (2018) Impact of chronic kidney disease on Watchman implantation: experience with 300 consecutive left atrial appendage closures at a single center. Heart Vessels 33: 1068-1075. [Crossref]

16. Fastner C, Brachmann J, Lewalter T, Zeymer U, Sievert H, et al. (2021) Left atrial appendage closure in patients with chronic kidney disease: results from the German multicentre LAARGE registry. Clin Res Cardiol 110: 12-20. [Crossref]

17. Kefer J, Tzikas A, Freixa X, Shakir S, Gafoor S, et al. (2016) Impact of chronic kidney disease on left atrial appendage occlusion for stroke prevention in patients with atrial fibrillation. Int J Cardiol 207: 335-340. [Crossref]

18. Aminian A, Lempereur M, Ben Yedder M, Dubois P (2018) Realtime fusion of echocardiography and fluoroscopy allowing successful implantation of a WATCHMAN device without contrast injection. EuroIntervention 14: 174-175. [Crossref]

19. Sedaghat A, Al-Kassou B, Vij V, Nelles D, Stuhr M, et al. (2019) Contrast-free, echocardiography- guided left atrial appendage occlusion (LAAo): a propensitymatched comparison with conventional LAAo using the AMPLATZER ${ }^{\mathrm{TM}}$ Amulet $^{\mathrm{TM}}$ device. Clin Res Cardiol 108: 333-340. [Crossref]

20. Italiano G, Maltagliati A, Mantegazza V, Fusini L, Mancini ME, et al. (2020) Multimodality approach for endovascular left atrial appendage closure: Head-tohead comparison among 2D and 3D echocardiography, angiography, and computer tomography. Diagnostics 10: E1103. [Crossref]

Copyright: (C2021 Abdirashid M. This is an open-access article distributed under the terms of the Creative Commons Attribution License, which permits unrestricted use, distribution, and reproduction in any medium, provided the original author and source are credited. 